\title{
Capacity Analysis of Wireless Powered Cooperative NOMA Networks over Generalized Fading
}

\author{
Orken Omarov, Galymzhan Nauryzbayev, ${ }^{\circ}$ Sultangali Arzykulov, Mohammad S. Hashmi, and ${ }^{\circ}$ Ahmed M. Eltawil \\ School of Engineering and Digital Sciences, Nazarbayev University, Nur-Sultan, Z05H0K3, Kazakhstan \\ ${ }^{\circ}$ Computer, Electrical, and Mathematical Sciences \& Engineering Division, KAUST, Thuwal, KSA 23955-6900 \\ Email: \{orken.omarov, galymzhan.nauryzbayev, mohammad.hashmi\}@nu.edu.kz, \\ $\circ\{$ sultangali.arzykulov, ahmed.eltawil\}@kaust.edu.sa
}

\begin{abstract}
This paper provides a performance evaluation of two-hop non-orthogonal multiple access (NOMA) architecture with energy harvesting (EH) cooperative agent and channel gains following the k-m fading, through analysis of ergodic capacity with respect to hardware impairments and channel conditions. The performance results of the distant user over two EH protocols, namely power splitting and time-switching relaying, are obtained and compared with simulation outcomes. The developed framework allows to evaluate the network under a range of external conditions and infers the importance of considering the hardware impairments.
\end{abstract}

Index Terms - Cooperative communications, ergodic capacity, simultaneous wireless information and power transfer (SWIPT), non-orthogonal multiple access (NOMA).

\section{INTRODUCTION}

The trends for high data rates, low latency and reliable communication have been raised over the last couple of years. According to the Cisco's recent report [1], the amount of traffic generated by wirelessly connected devices will increase to unprecedented rates, while the issue of bandwidth shortage is still present. A promising technique to enable such an architecture and provide spectral efficiency is considered to be non-orthogonal multiple access (NOMA) [2]. In contrast to its counterparts, NOMA allows us to use the same resource of wireless medium, i.e., time, frequency or code [3]. The research conducted on NOMA highlighted its advantages to enhance the overall network performance at the physical layer [4]. The authors in [5]-[7] studied the architecture where a cooperative NOMA network is considered.

In cooperative networks, the resources of cooperative agents are shared to create multiple replicas of the transmitted signal at the destination node [8]. Such a network architecture is widely used in applications, where the destination node's signal is subject to significant fading and the communicating nodes are equipped with single-antennas due to mobility, size and other constraints. Often, such applications ranging from Internet-of-Things to biomedical sensors experience energy limitations as well. This issue can be resolved by implementing wireless energy transfer methods.

One of the candidates to solve the energy limitation is simultaneous wireless information and power transfer (SWIPT). Although, it has not been implemented practically, the core idea of SWIPT was introduced in [9]. Two practical approaches for the energy harvesting (EH), namely, time-switching relaying (TSR) and power-splitting relaying (PSR) protocols, were introduced in [10], which became widely used approaches among the researchers. The PSR and TSR protocols operate through partitioning of the resources in the power and time domains, respectively. In particular, the authors in [11] provided some results on the performance estimation of a twohop cooperative NOMA network architecture over Weibull channels. However, more generalized fading such as an $\alpha-\mu$ statistical model was studied in [12]-[14] while omitting the impact of hardware imperfections. Due to the work in [15], the complementary metal-oxide-semiconductor (CMOS) technology enabled hardware operating with high-frequency signals, is subject to the imperfections. Thus, the detrimental effect of the hardware impairments is a significant issue to consider in the performance evaluation of wireless networks [16].

Hence, this paper analyzes the performance of a two-hop NOMA network, where the cooperative agent harvests the energy and relays the message in an amplify-and-forward (AF) mode. Moreover, the considered network incorporates the effect of hardware imperfections at the transceivers' radio frequency (RF) front-ends. The expressions of the ergodic capacity are derived for the abovementioned network. Using this performance metric, the effects of $\mathrm{EH}$ protocols and hardware impairments are evaluated over a wide range of channel conditions represented via a $\kappa-\mu$ general fading model.

\section{System AND ChANNEl Model}

We consider the cooperative NOMA network comprising a source $(S)$ and two receiver nodes, i.e., $U_{1}$ and $U_{2}$, (see Fig. 1), where $U_{1}$ operating in the $\mathrm{AF}$ relaying mode works as a cooperative agent. The network is a constellation of downlink half-duplex operated nodes with only one antenna for all purposes. Following the NOMA technique, $S$ broadcasts a composite message $x_{s}=\sum_{i=1}^{2} \sqrt{a_{i} P_{S}} x_{i}$, where $i, x_{i}$ and $a_{i}$ refer to the respective user, its message and power allocation factor, accordingly. For the analysis, $S$ transmits with a constant power of $P_{S}$ and the wireless channel conditions are assumed to be as $h_{1}>h_{2}$ due to their relative proximity and other factors. Therefore, following the NOMA approach, the relative power allocation factors are chosen as $a_{1}<a_{2}$. 
TABLE I. The variables in Eq. (7) for the PSR and TSR protocols.

\begin{tabular}{|c|c|c|c|c|c|}
\hline Variable & $n_{p}$ & $H_{p}$ & $R_{p}$ & $P_{p}^{R}$ & $G_{p}$ \\
\hline \hline PSR & $\sqrt{1-\rho} n_{1}+n_{c}$ & $1 / 2$ & $0.5 \varpi \rho T$ & $\varpi \rho P_{S} d_{1}^{-\tau}\left(1+\Omega_{s, u_{1}}^{2}\right)\left|h_{1}\right|^{2}$ & $\sqrt{\frac{\varpi \rho}{1-\rho}}$ \\
\hline TSR & $n_{1}$ & $(1-\eta) / 2$ & $\varpi \eta T$ & $\frac{2 \varpi \eta P_{S}\left(1+\Omega_{s, u_{1}}^{2}\right)}{(1-\eta) d_{1}^{\tau}}\left|h_{1}\right|^{2}$ & $\sqrt{\frac{2 \varpi \eta}{1-\eta}}$ \\
\hline
\end{tabular}

Due to the assumption that $U_{1}$ has better channel conditions, it acts as a cooperative agent to further amplify and forward the received message from $S$ to $U_{2}$. Thus, creating spatial diversity at the receiver side of $U_{2}$, which is considered as the main destination. Another assumption is that $U_{1}$ needs to harvest energy from the incoming electromagnetic signal to able to relay the message.

The distances related to the source-to-relay $\left(S\right.$-to- $\left.U_{1}\right)$, relayto-destination $\left(U_{1}\right.$-to- $\left.U_{2}\right)$ and source-to-destination $\left(S\right.$-to- $\left.U_{2}\right)$ links are denoted by $d_{1}, d_{2}$, and $d_{3}$, respectively. The corresponding channel fluctuations, denoted by $h_{1}, h_{2}$ and $h_{3}$, follow the $\kappa-\mu$ fading which is a generalized channel model able to emulate the Rayleigh $(\kappa \rightarrow 0$ and $\mu=1)$, Rice $(\kappa=k$ and $\mu=1$ ), etc. [17], [18]. The probability density function (PDF) of the $\kappa-\mu$ distribution is represented as follows

$$
f_{h_{i}}(r)=\frac{2 \mu_{i}\left(1+\kappa_{i}\right)^{\frac{\mu_{i}+1}{2}}}{e^{\mu_{i} \kappa_{i}} \kappa_{i}^{\frac{\mu_{i}-1}{2}}} r^{\mu_{i}} e^{-\phi_{i} r^{2}} I_{\mu_{i}-1}\left(2 \lambda_{i} r\right),
$$

where $\kappa>0$ is the ratio of line-of-sight and scattered signal wave powers, $\phi_{i}=\mu_{i}\left(1+\kappa_{i}\right)$ and $\lambda_{i}=\mu_{i} \sqrt{\kappa_{i}\left(1+\kappa_{i}\right)}$. The $I_{\nu}(\cdot)$ is the modified Bessel function of first kind and order $\nu$.

\section{Energy Harvesting Protocols}

Due to the broadcasting nature of communication, both $U_{1}$ and $U_{2}$ receive the superimposed NOMA signal directly as

$$
y_{i}=\sqrt{\frac{P_{S}}{d_{s, u_{i}}^{\tau}}} h_{s, u_{i}}\left(\sum_{j=1}^{2} \sqrt{a_{j}} x_{j}+\eta_{s, u_{i}}\right)+n_{i},
$$

where $\tau$ is the path-loss coefficient, $n_{i}$ is the the additive white Gaussian noise (AWGN) seen at the receiver node with the mean and variance being equal to zero and $\sigma_{i}^{2}$, respectively. Moreover, the combined hardware impairment level, denoted by $\eta_{s, u_{i}}$, follows the Complex Gaussian distribution with zero mean and variance of $\Omega_{s, u_{i}}^{2}$. According to [15], it is defined as $\Omega_{s, u_{i}} \triangleq \sqrt{\Omega_{s, t}^{2}+\Omega_{u_{i}, r}^{2}}$, considering the RF front-end imperfections of both transmitter and receiver. Moreover, $U_{2}$ receives the NOMA signal through direct path, and decodes message $x_{2}$ using the signal-to-interference-plus-distortionplus-noise ratio (SINDR) of

$$
\gamma_{U_{2}}^{\left\{x_{2}\right\}}=\frac{e_{1} Z}{e_{2} Z+e_{3}}
$$

where $Z=\left|h_{3}\right|^{2}, e_{1}=a_{2}, e_{2}=a_{1}+\Omega_{s-u_{2}}^{2}$, and $e_{3}=\frac{\sigma^{2} d_{3}^{\tau}}{P_{s}}$.

The whole transmission time block necessary for the message to reach its destination is denoted by $T$. In the PSR, $T$ is divided into 2 equal portions. The first time slot is dedicated to

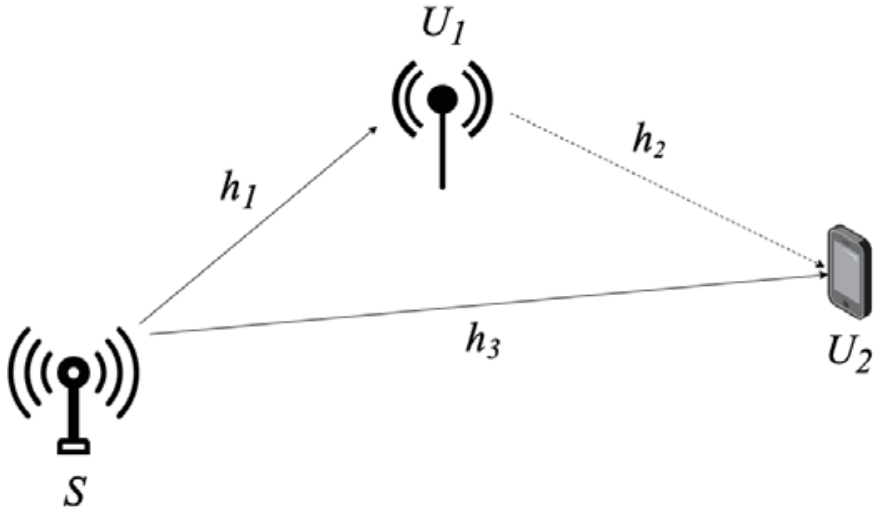

Fig. 1. A two-hop NOMA network with a cooperative agent.

the $S$-to- $U_{1}$ transmission, where $U_{1}$ harvests the $\rho P_{S}$ portion of power for further use and $(1-\rho) P_{S}$ portion of power is used for the information detection purpose. During the second time slot, $U_{1}$ uses the harvested energy to amplify and then forward the second user's signal. On the other hand, for the TSR case, $\eta T$ portion of the time $(0 \leq \eta \leq 1)$ is dedicated for the energy harvesting purposes and the rest of the time is split equally between the $S$-to- $U_{1}$ and $U_{1}$-to- $U_{2}$ transmissions.

For the PSR, the portion of the signal dedicated to the energy harvesting is written as

$$
\sqrt{\rho} y_{1}=\sqrt{\frac{\rho P_{S}}{d_{1}^{\tau}}} h_{1}\left(\sum_{j=1}^{2} \sqrt{a_{j}} x_{j}+\eta_{s, u_{1}}\right)+\sqrt{\rho} n_{1} .
$$

From this, the energy that can be harvested is further specified as

$$
E_{p}^{H}=R_{p}\left(P_{S}\left|h_{1}\right|^{2}\left(1+\Omega_{s, u_{1}}^{2}\right) d_{1}^{-\tau}+\sigma_{1}^{2}\right),
$$

where $0 \leq \varpi \leq 1$ is the harvesting efficiency and $p$ denotes the association of the variable either to the PSR or TSR protocol (See Table I), respectively. Furthermore, disregarding the little amount of an energy harvested from the noise term, Eq. (5) can be simplified and the expression of power for the next time slots is deduced as $P_{p}^{R}=\frac{E_{p}^{H}}{H_{p} T}$.

In the PSR protocol, the information detection is performed using a portion of the incoming signal, $y_{1}^{I T}=\sqrt{1-\rho} y_{1}+n_{c}$, where $n_{c}$ is the AWGN at the information receiver.

Following the NOMA principle, $U_{1}$ is capable of performing successive interference cancellation (SIC) by first decoding the stronger message $x_{2}$, and then its own message $x_{1}$ using 
TABLE II. The variables in Eqs. (6) and (8) for the PSR- and TSR-based protocols.

\begin{tabular}{|c|c|c|c|c|c|c|c|}
\hline Variable & $b_{1}$ & $b_{2}$ & $b_{3}$ & $c_{1}$ & $c_{2}$ & $c_{3}$ & $c_{4}$ \\
\hline \hline PSR & $a_{1}$ & $\Omega_{s-u_{1}}^{2}$ & $\frac{d_{1}^{\tau}}{P_{S}}\left(\sigma_{a}^{2}+\frac{\sigma_{c}^{2}}{1-\rho}\right)$ & $a_{2}$ & $a_{1}+\Omega_{s-u_{1}}^{2}$ & $\frac{d_{1}^{\tau}}{P_{S}}\left(\sigma_{a}^{2}+\frac{\sigma_{c}^{2}+\Omega_{u_{1}-u_{2}}^{2}}{(1-\rho)}\right)$ & $\frac{d_{1}^{\tau} d_{2}^{\tau} \sigma_{2}^{2}}{\varpi \rho P_{S}}$ \\
\hline $\mathrm{TSR}$ & $a_{1}$ & $\Omega_{s-u_{1}}^{2}$ & $\frac{d_{1}^{\tau} \sigma_{1}^{2}}{P_{S}}$ & $a_{2}$ & $a_{1}+\Omega_{s-u_{1}}^{2}$ & $\frac{d_{1}^{\tau}}{P_{S}}\left(\sigma_{1}^{2}+\Omega_{u_{1}-u_{2}}^{2}\right)$ & $\frac{\sigma_{2}^{2}}{P_{S}} \frac{d_{1}^{\tau} d_{2}^{\tau}(1-\eta)}{2 \varpi \eta}$ \\
\hline
\end{tabular}

signal-to-distortion-plus-noise ratio (SDNR) given by

$$
\gamma_{U_{1}}^{\left\{x_{1}\right\}}=\frac{b_{1} X}{b_{2} X+b_{3}},
$$

where $X=\left|h_{1}\right|^{2}$ and the rest parameters for both protocols are presented in Table II.

During the final transmission phase, $U_{1}$ uses the harvested energy and spends it on the amplification and forwarding the message towards $U_{2}$. The signal seen at $U_{2}$ is written as

$$
y_{1 \rightarrow 2}=\frac{G_{p} h_{2}}{\sqrt{d_{2}^{\tau}}}\left(D_{p}\left(y_{1}-n_{1}\right)+n_{p}+\eta_{u_{1}, u_{2}}\right)+n_{2},
$$

where $D_{p}$ is equal to $\sqrt{1-\rho}$ for the PSR and to 1 for the TSR, respectively. $n_{2}$ is the AWGN introduced at $U_{2}$ and $G_{p}$ is the amplification factor. Assuming the high signal-to-noise (SNR) values, the amplification factor can be simplified as $G_{p}=\sqrt{\frac{P_{p}^{R}}{W_{p}}}$, where $W_{p \rightarrow P S R}=(1-$ $\rho)\left(P_{s}\left(1+\Omega_{s, u_{1}}^{2}\right) d_{1}^{-\tau}\left|h_{1}\right|^{2}+\sigma_{1}^{2}\right)+\sigma_{c}^{2}$ and $W_{p \rightarrow T S R}=$ $\frac{\left(1+\Omega_{s, u_{1}}^{2}\right) P_{S}}{d_{1}^{\tau}}\left|h_{1}\right|^{2}+\sigma_{1}^{2}$ and $P_{p}^{R}$. Further, substituting the obtained gain expressions into Eq. (7), the SINDR for both protocols to decode $x_{2}$ is derived as

$$
\gamma_{U_{1 \rightarrow 2}}^{\left\{x_{2}\right\}}=\frac{c_{1} X Y}{c_{2} X Y+c_{3} Y+c_{4}},
$$

where $Y=\left|h_{2}\right|^{2}$ and $c_{1}, c_{2}, c_{3}$ and $c_{4}$ are shown in Table II.

\section{ERGODIC CAPACITY}

Proposition 1: For the $S$-to- $U_{2}$ direct link, the ergodic capacity expression is defined as in Eq. (9) for both protocols, at the top of the next page, where $E=e_{1}+e_{2}$ and $\alpha_{3}=\mu_{3}+q$.

Proof: See Appendix A.

Due to its complexity, the ergodic capacity evaluation for the exact SINDR expression of the $U_{1}$-to- $U_{2}$ link is not feasible. Thus, in the high-SNR regime (i.e., $c_{3} \rightarrow 0$ ), Eq. (8) can be approximated as

$$
\begin{aligned}
\gamma_{U_{1 \rightarrow 2}} & =\frac{c_{1} X Y}{Y c_{3}\left(\frac{c_{2}}{c_{3}} X+1\right)+c_{4}} \\
& \approx \frac{c_{1} X Y}{c_{2} X Y+c_{4}} .
\end{aligned}
$$

Proposition 2: For the $U_{1}$-to- $U_{2}$ link, the ergodic capacity expression is defined as in Eq. (10) for both protocols, where $c_{5}=c_{1}+c_{2}, \beta_{1}=\frac{\mu_{2}+\mu_{1}+l+q}{2}$ and $\beta_{2}=\frac{\mu_{2}-\mu_{1}+l-q}{2}$.

Proof: See Appendix B.

Additionally, the asymptotic characteristic of the $U_{1}$-to- $U_{2}$ link capacity is evaluated to be explicit. As a result, the SINDR

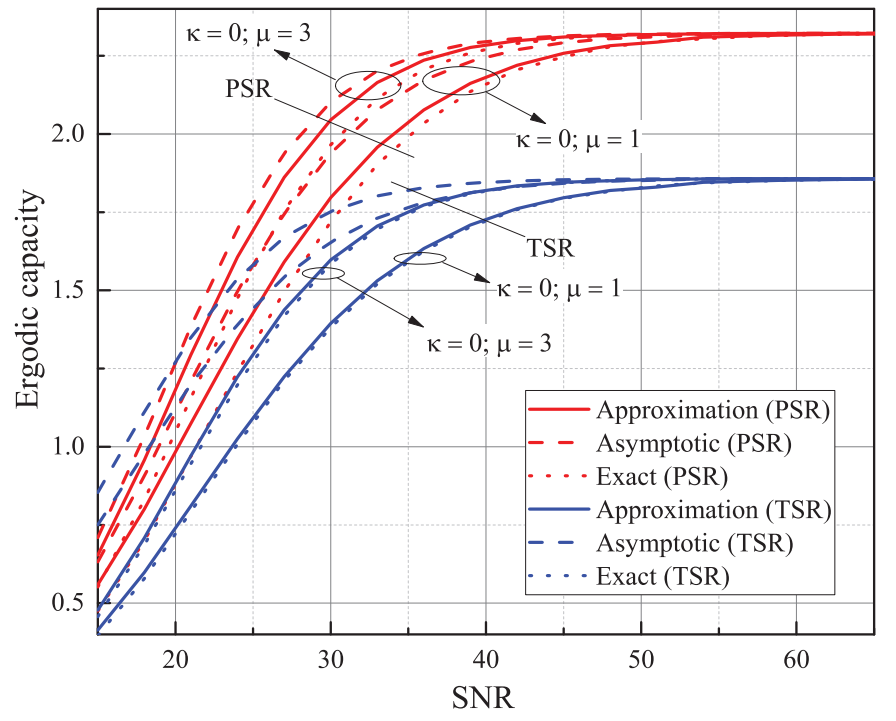

Fig. 2. The exact, approximate and asymptotic values of ergodic capacity for the $\operatorname{TSR}(\eta=0.2)$ and $\operatorname{PSR}(\rho=0.7)$ protocols, when $\kappa=0, \mu=\{1,3\}$ and $\Omega=0$.

at $U_{2}$ is changed to

$$
\gamma_{U_{1 \rightarrow 2}}=\frac{c_{1} X}{c_{2} X+c_{3}}
$$

accounting for $c_{4} \rightarrow 0$. Due to the similarity of the general form of asymptotic SINDR with the previously mentioned case of direct transmission, the explanations are omitted here. The asymptotic expression defining the average capacity for the $U_{1}$-to- $U_{2}$ link is shown in Eq. (11), where $\alpha_{1}=\mu_{1}+q$. Then, the overall ergodic capacity at $U_{2}$ over the whole transmission period is expressed as

$$
C_{e r g}^{U_{2}}=C_{e r g}^{\gamma_{U_{1} \rightarrow 2}}+C_{e r g}^{\gamma_{U_{2}}} .
$$

\section{NUMERICAL RESULTS}

This section describes the obtained results for the ergodic capacity expressions and their respective comparison with the Monte-Carlo simulations. For this analysis, the distances are set as $d_{1}=2 \mathrm{~m}^{1}, d_{2}=1.3 \mathrm{~m}$, and $d_{3}=3 \mathrm{~m}$. In addition, we set $\varpi=0.6, \tau=3, a_{1}=0.2$ and $a_{2}=0.8$, [19], [20].

Fig. 2 illustrates the change of ergodic capacity due to different channel conditions for both protocols, while assuming the perfect hardware impairments scenario. For the whole range of SNR values and respective conditions, the

\footnotetext{
${ }^{1}$ Due to the relative proximity of $U_{1}$ to $S$, it is assumed that the performance level of this link is sufficient to support its quality of service.
} 


$$
\begin{array}{r}
C_{e r g}^{\gamma_{U_{2}}}=\frac{H_{p} \psi_{3}}{\ln (2)} \sum_{q=0}^{\infty} \frac{\left[\lambda_{3}\right]^{\mu_{3}-1+2 q}}{\Gamma\left(\alpha_{3}\right) q !}\left[\left(\frac{e_{3}}{E}\right)^{\alpha_{3}} G_{2,3}^{3,1}\left(\frac{\phi_{3} e_{3}}{E} \mid \begin{array}{c}
-\alpha_{3}, 1-\alpha_{3} \\
0,-\alpha_{3},-\alpha_{3}
\end{array}\right)-\left(\frac{e_{3}}{e_{2}}\right)^{\alpha_{3}} G_{2,3}^{3,1}\left(\frac{\phi_{3} e_{3}}{e_{2}} \mid \begin{array}{c}
-\alpha_{3}, 1-\alpha_{3} \\
0,-\alpha_{3},-\alpha_{3}
\end{array}\right)\right] \\
C_{e r g, A p r}^{\gamma_{U_{1} \rightarrow 2}}=\frac{H_{p} \psi_{1} \psi_{2}}{\ln (2)} \sum_{q=0}^{\infty} \frac{\lambda_{1}^{\mu_{1}-1+2 q}}{\Gamma\left(\alpha_{1}\right) q !} \sum_{l=0}^{\infty} \frac{\lambda_{2}^{\mu_{2}-1+2 l} c_{4}^{\beta_{1}}}{\Gamma\left(\mu_{2}+l\right) l !}\left(\frac{\phi_{1}}{\phi_{2}}\right)^{\beta_{2}}\left(\left(\frac{1}{c_{5}}\right)^{\alpha_{1}+\beta_{2}} G_{2,4}^{4,1}\left(\frac{\phi_{1} \phi_{2} c_{4}}{c_{5}} \mid \begin{array}{c}
-\beta_{1}, 1-\beta_{1} \\
\beta_{2},-\beta_{2},-\beta_{1},-\beta_{1}
\end{array}\right)\right. \\
\left.-\left(\frac{1}{c_{2}}\right)^{\alpha_{1}+\beta_{2}} G_{2,4}^{4,1}\left(\frac{\phi_{1} \phi_{2} c_{4}}{c_{2}} \mid \begin{array}{c}
-\beta_{1}, 1-\beta_{1} \\
\beta_{2},-\beta_{2},-\beta_{1},-\beta_{1}
\end{array}\right)\right) \\
C_{e r g, A s m}^{\gamma_{U_{1} \rightarrow 2}}=\frac{H_{p} \psi_{1}}{\ln (2)} \sum_{q=0}^{\infty} \frac{\lambda_{1}^{\mu_{1}-1+2 q}}{\Gamma\left(\alpha_{1}\right) q !}\left[\left(\frac{c_{3}}{c_{5}}\right)^{\alpha_{1}} G_{2,3}^{3,1}\left(\frac{\phi_{1} c_{3}}{c_{5}} \mid \underset{0,-\alpha_{1}, 1-\alpha_{1}}{0,-\alpha_{1}}\right)-\left(\frac{c_{3}}{c_{2}}\right)^{\alpha_{1}} G_{2,3}^{3,1}\left(\frac{\phi_{1} c_{3}}{c_{2}} \mid \begin{array}{c}
-\alpha_{1}, 1-\alpha_{1} \\
0,-\alpha_{1},-\alpha_{1}
\end{array}\right)\right]
\end{array}
$$

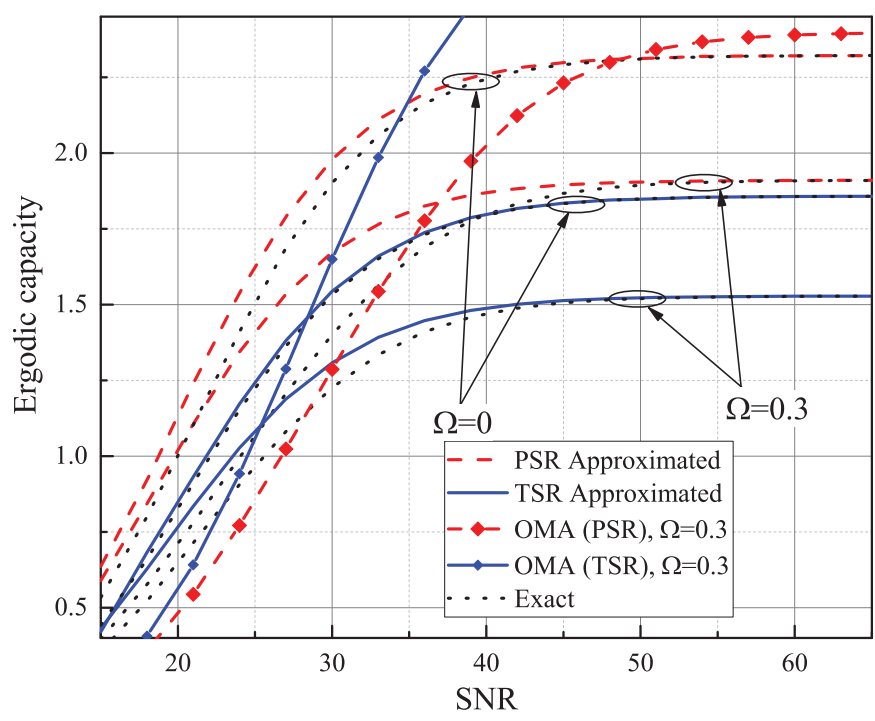

Fig. 3. The ergodic capacity for the TSR $(\eta=0.2)$ and PSR ( $\rho=0.7)$ protocols using NOMA and OMA techniques, when $\Omega=\{0,0.3\}, \kappa=3$ and $\mu=1$.

PSR protocol shows a better performance compared to the TSR. Increasing the number of multipath clusters $(\mu)$ improves the overall performance of both protocols. The saturation for both protocols can be explained by the NOMA's constraints. Overall, the approximation results follow closely the exact values for both TSR and PSR protocols, while a slightly better correlation is observed for the TSR case.

Similarly, Fig. 3 shows the effect of different hardware impairment values on the average capacity over Rician channels. In all of the cases below, the Rician $K$ parameter signifying the ratio of in-phase and quadrature-phase signals is defined to be 3 . For both protocols, a significant degradation of the capacity performance is observed due to the increase in hardware impairments. For example, in the PSR, the average capacity at $50 \mathrm{~dB}$ is around $2.3 \mathrm{bits} / \mathrm{s} / \mathrm{Hz}$ for the case with no hardware impairments, while it approaches $1.87 \mathrm{bits} / \mathrm{s} / \mathrm{Hz}$, when

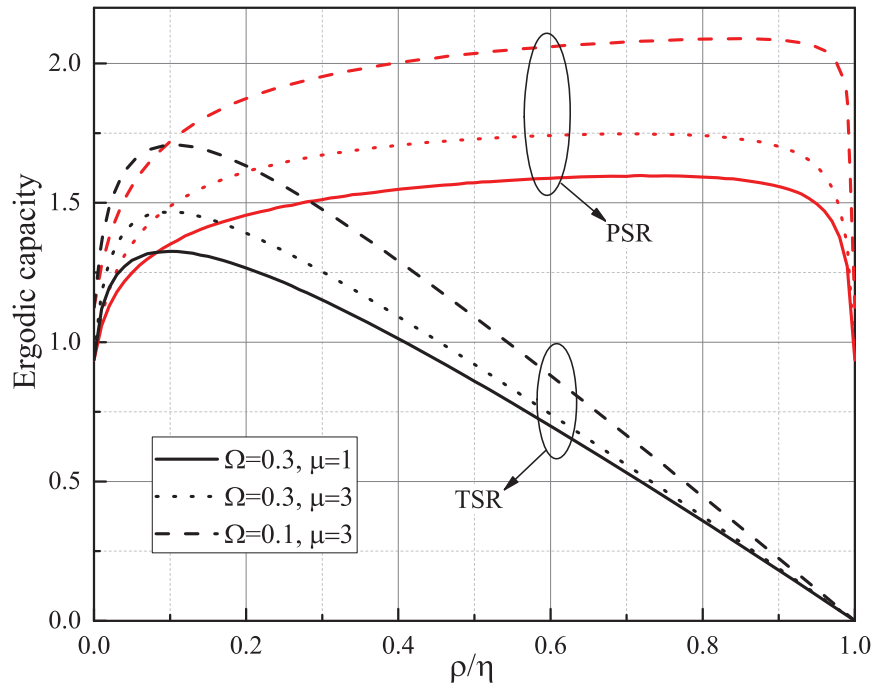

Fig. 4. The ergodic capacity versus $\rho / \eta$ for the TSR and PSR at $50 \mathrm{~dB}$ for various $\Omega$ and $\mu$ values when $\kappa=0$.

impairments are introduced. Additionally, for comparison with the OMA benchmark, the capacity plots are shown, where the saturation starts after $40 \mathrm{~dB}$ due to the hardware impairments. In essence, all NOMA cases start saturating at $30 \mathrm{~dB}$ under any hardware impairments level.

Fig. 4 presents the simulation results for the performance change under a set of energy harvesting factors $\rho$ and $\eta$ at 50 $\mathrm{dB}$. As in the previous cases, the metric shows better results under the PSR rather than the TSR. For both PSR and TSR cases, the effect of hardware impairments is seen as more drastic, when compared with the change of $\mu$ values. When the portion of the power allocated for the energy harvesting is small or too much, the performance degrades. The optimal energy harvesting factors for the TSR and PSR are defined as 0.2 and 0.9 , respectively. These values hold true when the external factors affecting the network are changed. 


\section{CONCLUSION}

This paper analyses the ergodic capacity metric of the distant user in a cooperative two-hop NOMA network, while considering the energy-constrained cooperative agent with hardware impairments and channel gains following the $\kappa-\mu$ fading model. The approximate and asymptotic expressions were derived and examined over the PSR and TSR protocols. The obtained results demonstrated the importance of the hardware impairments, as they tend to significantly affect the overall performance. The accomplished work serves as a framework to foster further research in the area, as it contributes by delivering a general expression for the performance evaluation under a set of channel statistics and transceiver hardware quality.

\section{ACKNOWLEDGMENT}

This work was supported by the Nazarbayev University Faculty Development Competitive Research Program under Grant no. 240919FD3935.

\section{APPENDIX A}

\section{DERIVATION OF PROPOSITION 1}

For the $S$-to- $U_{2}$ direct link, the ergodic capacity expression is defined the following way

$$
\begin{aligned}
C_{\text {erg }}^{\gamma_{U_{2}}}= & \int_{0}^{\infty} H_{p} \log _{2}\left(1+\gamma_{U_{2}}\right) f_{\gamma_{U_{2}}} \mathrm{~d} \gamma_{U_{2}} \\
= & \int_{0}^{\infty} H_{p} \log _{2}\left(e_{3}+\left(e_{1}+e_{2}\right) Z\right) f_{G}(g) \mathrm{d} g \\
& -\int_{0}^{\infty} H_{p} \log _{2}\left(e_{3}+e_{2} Z\right) f_{Q}(q) \mathrm{d} q \\
= & \frac{H_{p}}{\ln (2)}\left[\int_{0}^{\infty} \ln \left(1+\frac{g}{e_{3}}\right) f_{G}(g) \mathrm{d} g\right. \\
& \left.-\int_{0}^{\infty} \ln \left(1+\frac{q}{e_{3}}\right) f_{Q}(q) \mathrm{d} q\right],
\end{aligned}
$$

where $G=E Z$ and $Q=e_{2} Z$ and their probability density functions are defined as $f_{G}(g)=\frac{1}{E} f_{Z}\left(\frac{g}{E}\right)$ and $f_{Q}(q)=$ $\frac{1}{e_{2}} f_{Z}\left(\frac{q}{e_{2}}\right)$, respectively. Using the "change of variable" method [23] for $Z=\left|h_{3}\right|^{2}$, and defining $\psi=\frac{\mu(1+\kappa) \frac{\mu+1}{2}}{e^{\mu \kappa} \kappa \frac{\mu-1}{2}}$, the PDF of $Z$ can be written as

$$
f_{Z}(z)=\psi z^{\frac{\mu-1}{2}} e^{-\phi z} I_{\mu-1}(2 \lambda \sqrt{z}) .
$$

Noticing the similarity in a structure of $f_{G}(g)$ and $f_{Q}(q)$, and using the infinite sum representation of the Bessel function, we write

$$
f_{G}(g)=\sum_{q=0}^{\infty} \frac{\lambda_{3}^{\mu_{3}-1+2 q}}{\Gamma\left(\alpha_{3}\right) q !} \psi_{3}\left(\frac{1}{E}\right)^{\alpha_{3}} g^{\alpha_{3}-1} e^{\left(\frac{-\phi_{3} g}{E}\right)} .
$$

As part of the capacity derivation process, using the following replacements

$$
\begin{gathered}
\ln (1+a)=G_{2,2}^{1,2}\left(a \mid \begin{array}{c}
1,1 \\
1,0
\end{array}\right), \\
e^{-a}=G_{0,1}^{1,0}\left(a \mid \begin{array}{c}
\overline{0}
\end{array}\right),
\end{gathered}
$$

and the results presented in [22, Eq. (21)], the following is true

$$
\begin{aligned}
& \int_{0}^{\infty} \ln \left(1+\frac{g}{e_{3}}\right) f_{G}(g) \mathrm{d} g=\sum_{q=0}^{\infty} \frac{\left[\lambda_{3}\right]^{\mu_{3}-1+2 q}}{\Gamma\left(\alpha_{3}\right) q !} \psi_{3}\left(\frac{e_{3}}{E}\right)^{\alpha_{3}} \\
& \times G_{2,3}^{3,1}\left(\frac{\phi_{3} e_{3}}{E} \mid \begin{array}{c}
-\alpha_{3}, 1-\alpha_{3} \\
0,-\alpha_{3},-\alpha_{3}
\end{array}\right) . \quad \text { A }
\end{aligned}
$$

Extrapolating the obtained results to the case of variable $q$ and further substituting them gives the general ergodic capacity expression shown as in Eq. (9).

\section{APPENDIX B}

\section{DERIVATION OF PROPOSITION 2}

The capacity expression for the $U_{1}$-to- $U_{2}$ link is given by

$$
\begin{aligned}
C_{e r g}= & \int_{0}^{\infty} H_{p} \log _{2}\left(1+\gamma_{U_{1 \rightarrow 2}}\right) f_{\gamma_{U_{1 \rightarrow 2}}}\left(\gamma_{U_{1 \rightarrow 2}}\right) \mathrm{d} \gamma_{U_{1 \rightarrow 2}} \\
= & \int_{0}^{\infty} H_{p} \log _{2}\left(c_{4}+\left(c_{1}+c_{2}\right) X Y\right) f_{T}(t) \mathrm{d} t \\
& -\int_{0}^{\infty} H_{p} \log _{2}\left(c_{4}+c_{2} X Y\right) f_{V}(v) \mathrm{d} v \\
= & \frac{H_{p}}{\ln (2)}\left[\int_{0}^{\infty} \ln \left(1+\frac{t}{c_{4}}\right) f_{T}(t) \mathrm{d} t\right. \\
& \left.-\int_{0}^{\infty} \ln \left(1+\frac{v}{c_{4}}\right) f_{V}(v) \mathrm{d} v\right]
\end{aligned}
$$

where $T=c_{5} X Y$ and $V=c_{2} X Y$.

The new density function is obtained as

$$
f_{T}(t)=\int_{0}^{\infty} \frac{1}{y c_{5}} f_{Y}(y) f_{X}\left(\frac{t}{y c_{5}}\right) \mathrm{d} y
$$

Due to their similarity, the thorough derivation of the PDF is shown only for variable $t$. Re-expressing the Bessel function in terms of infinite sum and using [21, Eq. 3.478.4], the new PDF is written as

$$
\begin{aligned}
f_{T}(t)= & \sum_{q=0}^{\infty} \frac{2 \psi_{1} \psi_{2} \lambda_{1}^{\mu_{1}-1+2 q}}{\Gamma\left(\alpha_{1}\right) q !} \sum_{l=0}^{\infty} \frac{\lambda_{2}^{\mu_{2}-1+2 l} t^{-1}}{\Gamma\left(\mu_{2}+l\right) l !} \\
& \times\left(\frac{t}{c_{5}}\right)^{\alpha_{1}+\beta_{2}}\left(\frac{\phi_{1}}{\phi_{2}}\right)^{\beta_{2}} K_{2 \beta_{2}}\left(2 \sqrt{\frac{\phi_{1} \phi_{2} t}{c_{5}}}\right) .
\end{aligned}
$$

In the same manner, as part of the capacity derivation process, using the $K_{\nu}(a)=\frac{1}{2} G_{0,2}^{2,0}\left(\frac{a^{2}}{4} \mid \frac{\dot{\nu}}{2},-\frac{i}{2}\right)$ as a replacement, we can further write

$$
\begin{gathered}
\int_{0}^{\infty} \ln \left(1+\frac{t}{c_{4}}\right) f_{T}(t) \mathrm{d} t=\sum_{q=0}^{\infty} \sum_{l=0}^{\infty} \frac{\psi_{1} \psi_{2} \lambda_{1}^{\mu_{1}-1+2 q}}{\Gamma\left(\alpha_{1}\right) q ! c_{5}^{\alpha_{1}+\beta_{2}}}\left(\frac{\phi_{1}}{\phi_{2}}\right)^{\beta_{2}} \\
\times \frac{\lambda_{2}^{\mu_{2}-1+2 l} c_{4}^{\beta_{1}}}{\Gamma\left(\mu_{2}+l\right) l !} G_{2,4}^{4,1}\left(\frac{\phi_{1} \phi_{2} c_{4}}{c_{5}} \mid \begin{array}{c}
-\beta_{1}, 1-\beta_{1} \\
\beta_{2},-\beta_{2},-\beta_{1},-\beta_{1}
\end{array}\right) .
\end{gathered}
$$

Moreover, applying the results presented in [22, Eq. (21)], the integral in Eq. (B.4) is solved. Extrapolating the obtained results to the case of $v$ and further substituting them, gives the general ergodic capacity expression, as shown in Eq. (10). 


\section{REFERENCES}

[1] Cisco, "Annual Internet Report 2018-2023 (white paper)," CISCO, Tech. Rep., Updated on Mar. 2020.

[2] Y. Saito et al., "Non-Orthogonal Multiple Access (NOMA) for Cellular Future Radio Access," IEEE 77th Vehicular Technology Conference (VTC Spring), Dresden, 2013, pp. 1-5.

[3] Z. Ding et al., "A Survey on Non-Orthogonal Multiple Access for 5G Networks: Research Challenges and Future Trends," Journal on Selected Areas in Communications, vol. 35, no. 10, pp. 2181-2195, October 2017.

[4] Z. Ding et al., "On the Performance of Non-Orthogonal Multiple Access in 5G Systems with Randomly Deployed Users," IEEE Signal Processing Letters, vol. 21, no. 12, pp. 1501-1505, December 2014.

[5] Z. Ding et al., "Cooperative Non-Orthogonal Multiple Access in 5G Systems," IEEE Communications Letters, vol. 19, no. 8, pp. 1462-1465, August 2015.

[6] Y. Liu et al., "Cooperative non-orthogonal multiple access with simultaneous wireless information and power transfer," Journal on Selected Areas in Communications, vol. 34, no. 4, pp. 938-953, April 2016.

[7] O. Omarov et al., "Hardware-Limited Cooperative SWIPT-enabled NOMA Networks," 12th IEEE/IET International Symposium on Communication Systems, Networks and Digital Signal Processing (CSNDSP), Porto, Portugal, 2020, pp. 1-6.

[8] A. Nosratinia et al., "Cooperative communication in wireless networks," IEEE Communications Magazine, vol. 42, no. 10, pp. 74-80, October 2004.

[9] L. R. Varshney, "Transporting information and energy simultaneously," IEEE International Symposium on Information Theory, Toronto, ON, 2008, pp. 1612-1616.

[10] A. A. Nasir et al., "Relaying Protocols for Wireless Energy Harvesting and Information Processing," IEEE Transactions on Wireless Communications, vol. 12, no. 7, pp. 3622-3636, July 2013.

[11] X. Li et al., "Performance Analysis of Impaired SWIPT NOMA Relaying Networks Over Imperfect Weibull Channels," IEEE Systems Journal, vol. 14 , no. 1, pp. 669-672, March 2020.

[12] K. Dautov et al., "On the Performance of UAV-enabled Multihop V2V FSO systems over generalized $\alpha-\mu$ Channels," International
Conference on Computing and Network Communications (CoCoNet), Astana, 2018, pp. 69-73.

[13] G. Nauryzbayev, M. Abdallah, and K. M. Rabie, "Outage Probability of the EH-Based Full-Duplex AF and DF Relaying Systems in $\alpha-\mu$ Environment," IEEE 88th Vehicular Technology Conference (VTC-Fall), Chicago, IL, USA, 2018, pp. 1-6.

[14] G. Nauryzbayev et al., "Underlay CR-NOMA Relaying Networks over Non-Homogeneous Generalized Fading Channels," 12th IEEE/IET CSNDSP, Porto, Portugal, 2020, pp. 1-6.

[15] T. Schenk, RF Imperfections in High-rate Wireless Systems: Impact and Digital Compensation. Dordrecht, The Netherlands: Springer, 2008.

[16] S. Arzykulov et al., "Hardware- and Interference-Limited Cognitive IoT Relaying NOMA Networks With Imperfect SIC Over Generalized NonHomogeneous Fading Channels," IEEE Access, vol. 8, pp. 72942-72956, 2020.

[17] M. D. Yacoub, "The $\kappa-\mu$ distribution and the $\eta-\mu$ distribution," IEEE Antennas and Propagation Magazine, vol. 49, no. 1, pp. 68-81, February 2007.

[18] K. Rabie et al., "Full-Duplex Energy-Harvesting Enabled Relay Networks in Generalized Fading Channels," IEEE Wireless Communications Letters, vol. 8, no. 2, pp. 384-387, April 2019.

[19] C. R. Valenta and G. D. Durgin, "Harvesting Wireless Power: Survey of Energy-Harvester Conversion Efficiency in Far-Field, Wireless Power Transfer Systems," IEEE Microwave Magazine, vol. 15, no. 4, pp. 108120, June 2014

[20] V. Erceg et al., "An empirically based path loss model for wireless channels in suburban environments," IEEE Journal on Selected Areas in Communications, vol. 17, no. 7, pp. 1205-1211, July 1999.

[21] I. S. Gradshteyn and I. M. Ryzhik, Table of Integrals, Series, and Products. San Diego, CA, USA: Academic Press, 2007.

[22] V. S. Adamchik O. I. Marichev "The algorithm for calculating integrals of hypergeometric type functions and its realization in REDUCE system," International Symposium on Symbolic and Algebraic Computation (ISSAC), Tokyo, Japan, pp. 212-224, August 1990.

[23] G. Nauryzbayev et al., "On the Performance Analysis of WPT-Based Dual-Hop AF Relaying Networks in $\alpha-\mu$ Fading," IEEE Access, vol. 6, pp. 37138-37149, 2018. 